\title{
A Semiotic Approach to Quality in Requirements Specifications
}

John Krogstie

SINTEF Telecom and Informatics and IDI, NTNU, Forskningsveien 1, N-0314 Oslo, Norway, Phone: +47 22067425, Fax: +47 22067350, Email: John.Krogstie@informatics.sintef.no

\begin{abstract}
We have earlier developed a framework for understanding and assessing the quality of models in general, with emphasis on models made in conceptual modeling languages. At the same time, there is a long tradition on discussing quality of requirements specifications. Several authors have created taxonomies of useful properties of models and requirements specifications, as summarized by Alan Davis in his article from 1993. We have in this paper extended our quality framework for models based on the work by Davis on quality in requirement specifications, looking upon a requirements specification as a specific type of model. Comparing the approaches we find on the one hand that the properties as summarized by Davis are subsumed by our framework on a high level, and that there are aspects within our framework that are not covered by Davis. On the other hand, the comparison has resulted in a useful deepening of our framework on this specific kind of model, and in this way improved the practical applicability of our framework when applied to discussing the quality of requirements specifications.
\end{abstract}

Key words: Requirements specification, Conceptual modeling, Quality

\section{INTRODUCTION}

Modelling activities are central in the development and maintenance of computerised information systems. Examples of types of models that are developed are:

- The existing information systems in the organisation as they are perceived by the participants of the project are modelled during analysis.

The original version of this chapter was revised: The copyright line was incorrect. This has been corrected. The Erratum to this chapter is available at DOI: 10.1007/978-0-387-35611-2_22 
- The future information system (IS) is modelled during requirement specification.

- The future computerised information system (CIS) is modelled during design.

Most proposals for quality goals for requirements models as summarised in (Davis et al. 1993) have included many useful aspects, but unfortunately mostly in the form of unsystematic lists of desired properties. Some researchers (Falkenberg 1996; Lindland Sindre and Sølvberg 1994; Pohl 1994) have attempted to take a more structured approach for understanding quality. This has culminated in the work presented in (Krogstie, Lindland and Sindre 1995a; Krogstie, Lindland and Sindre 1995b; Krogstie and Sølvberg 2000; Krogstie 2001), and we will in this paper integrated this framework with the work of Davis et al, and in this way extend the framework in the area of requirements models.

The paper is structured as follows. In the next section, the quality framework is presented briefly. In Section 3, we have specialised the framework based on the quality properties for a software requirements specification (SRS) as presented by Davis. A summary of the results is presented in section 4 , before ending the paper pointing to future work.

\section{FRAMEWORK FOR QUALITY OF MODELS}

The framework has several distinguishing properties:

- It is closely linked to linguistic and semiotic concepts because we recognise that modelling is essentially making statements in some language. In particular, the core of the framework including the discussion on syntax, semantics, and pragmatics is parallel to the use of these terms in the semiotic theory of Morris (see e.g. (Nöth 1990)).

- It is based on a constructivistic world-view (Berger and Luckmann 1966), recognising that models are usually created as part of a dialogue between the participants involved in modelling, whose knowledge of the modelling domain changes as modelling takes place, and where the domain is also developed (socially (re)constructed) as a side-effect of the modelling activity.

- It distinguishes between goals and means that support the achievement of the goals. Even if it can be argued from both activity theory (Engeström 1999) and decision theory (Schuette 1999) that the interrelationships between goals and means are being determined through the preference function of the modeller applied at the modelling task at hand, we have found that most modelling techniques in practice can be found to primarily contribute to a specific model quality goal. 


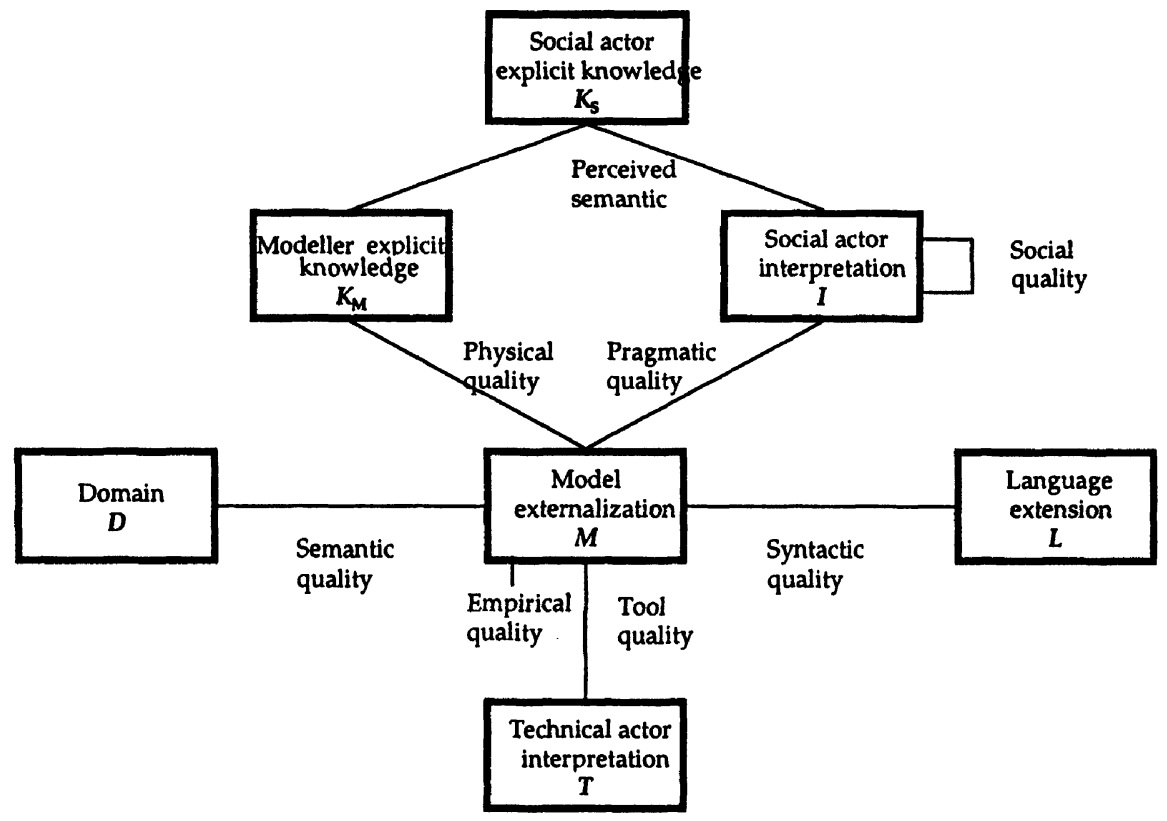

Figure 1. Main parts of the quality framework

The main concepts of the framework and their relationships are shown in the Figure 1. We have taken a set-theoretic approach to the discussion of model quality at different semiotic levels, which has been defined as the correspondence between statements belonging to the following sets:

- $\boldsymbol{L}$, the language extension, i.e., the set of all statements that are possible to make according to the graphemes, vocabulary, and syntax of the modelling languages used. Note that we have here also included the structuring rules of a natural language document. Some work on natural language requirement specification handle structure as a separate area (Fabbrini et al. 1998).

- $D$, the domain, i.e., the set of all statements which can be stated about the situation at hand. Note that the domains we are normally dealing with in information systems development has been socially constructed, and are more or less inter-subjectively agreed. That the world is socially constructed does not make it any less important to model that world (Dahlbom 1991), to be able to understand and potentially reconstruct it.

- $M$, the externalised model, i.e. the set of all statements in someone's model of part of the perceived reality written in a language.

- $K_{s}$, the relevant explicit knowledge of the stakeholders being involved in modelling (the audience $A$ ). A subset of the audience is those actually actively involved in modelling, and their knowledge is indicated by $K_{M}$.

- $I$, the social actor interpretation, i.e. the set of all statements which the audience think that an externalised model consists of. 
- $T$, the technical actor interpretation, i.e. the statements in the model as 'interpreted' by the different tools used in modelling.

The main quality types are indicated by solid lines between the sets, and are described briefly below, following the levels of a semiotic ladder as described in (Falkenberg et al. 1996).

- Physical quality: The basic quality goals on the physical level is externalisation, that the knowledge $\boldsymbol{K}$ of the domain $\boldsymbol{D}$ of some social actor has been externalised by the use of a modeling language, and internaliseability, that the externalised model $\boldsymbol{M}$ is persistent and available enabling the audience to make sense of it.

- Empirical quality: deals with predicable error frequencies when a model is read or written by different users, coding (e.g. shapes of boxes) and ergonomics of computer-human interaction for documentation and modelling-tools.

- Syntactic quality is the correspondence between the model $\boldsymbol{M}$ and the language extension $L$ of the language in which the model is written. This includes aspects of lexicon correctness, syntax correctness and structural quality (Fabbrini et al. 1998).

- Semantic quality is the correspondence between the model $M$ and the domain $\boldsymbol{D}$. The framework contains two semantic goals; Validity which means that all statements made in the model are correct relative to the domain and completeness which means that the model contains all the statements which is found in the domain. These goals are made more applicable by introducing the notion of feasibility relative to the purpose of modelling.

- Perceived semantic quality is the similar correspondence between the participants interpretation $I$ of a model $M$ and his or hers current knowledge $K$ of the domain $D$. Whereas the primary goal for semantic quality is a correspondence between the externalised model and the domain as indicated above, this correspondence can neither be established nor checked directly. To build a model, one has to go through the participant's knowledge regarding the domain, and to check the model, one has to compare with the participant's interpretation of the externalised model. Hence, what we observe at quality control is not the semantic quality of the model, but a perceived semantic quality based on comparisons of the two interpretations.

- Pragmatic quality is the correspondence between the model $\boldsymbol{M}$ and the audience's interpretation of it $(I)$.

- Finally, the goal defined for social quality is agreement among participants' interpretations $I$.

Goals and means to achieve these goals being model and language properties and modelling activities have been identified, and we will return 
to these in more detail throughout the paper. One of these means is the use of appropriate modelling languages. In the framework, language quality is divided into five areas:

1. Domain appropriateness. This relates the language to the domain and vice versa. The main goal is that there are no statements in the domain that cannot be expressed in the language. Obviously, different modelling languages are more or less suitable for different problem situations. Ideally, the conceptual basis must be powerful enough to express anything in the domain, not having what (Wand and Weber 1993) terms construct deficit. On the other hand you should not be able to express things that are not in the domain, if this makes you focus on the wrong aspects, i.e. what is termed construct excess (Wand and Weber 1993).

2. Participant language knowledge appropriateness relates the participant knowledge to the language. The overall goal here is that all the statements in the language models of the languages used by the different participants are part of the explicit knowledge of this participant. On the other hand, the knowledge of the participants is not static; i.e. it is possible to educate persons in the use of a specific language. In that case should one base the language on experience with languages that have been used successfully earlier on similar tasks.

3. Knowledge externalisability appropriateness relates the language to the participant knowledge. The goal is that there are no statements in the explicit knowledge of the participant that can not be expressed in the language. This focuses on how relevant knowledge may be articulated in the modelling language.

4. Participant comprehensibility appropriateness relates the language to the social actor interpretation. The idealised goal is that the participants in the modelling effort using the language understand all the possible statements of the language.

5. Technical actor interpretation appropriateness relates the language to the technical audience interpretations. For the technical actors (tools), it is especially important that the language lend itself to automatic reasoning. This requires formality (i.e. both formal syntax and semantics being operational and/or logical), but formality is not necessarily enough, since the reasoning must also be efficient to be of practical use. This is covered by what we term analysability (to exploit any mathematical semantics of the language) and executability (to exploit any operational semantics). 
Table 1. Goals and means in the quality framework

\begin{tabular}{|c|c|c|c|}
\hline $\begin{array}{l}\text { Quality } \\
\text { type }\end{array}$ & Goals & $\begin{array}{l}\text { Means } \\
\text { Model and language } \\
\text { properties }\end{array}$ & $\begin{array}{l}\text { Activities and } \\
\text { tool-support }\end{array}$ \\
\hline \multirow[t]{2}{*}{ Physical } & Externalization & $\begin{array}{l}\text { Domain appropriateness } \\
\text { Participant language } \\
\text { knowledge } \\
\text { appropriateness } \\
\text { Knowledge } \\
\text { externalizeability } \\
\text { appropriateness } \\
\text { Language extension } \\
\text { mechanism }\end{array}$ & $\begin{array}{l}\text { Meta-model } \\
\text { adaptation } \\
\text { Language extension } \\
\text { Statement insertion } \\
\text { Statement deletion }\end{array}$ \\
\hline & Internalizeability & $\begin{array}{l}\text { Persistence } \\
\text { Availability }\end{array}$ & $\begin{array}{l}\text { Database activities } \\
\text { Repository } \\
\text { functionality }\end{array}$ \\
\hline Empirical & $\begin{array}{l}\text { Minimal error } \\
\text { frequency }\end{array}$ & $\begin{array}{l}\text { Comprehensibility } \\
\text { appropriateness } \\
\text { Aesthetics }\end{array}$ & $\begin{array}{l}\text { Diagram layout } \\
\text { Readability index }\end{array}$ \\
\hline Syntactic & $\begin{array}{l}\text { Syntactic } \\
\text { correctness }\end{array}$ & Formal syntax & $\begin{array}{l}\text { Error prevention } \\
\text { Error detection } \\
\text { Error correction }\end{array}$ \\
\hline Semantic & $\begin{array}{l}\text { Feasible validity } \\
\text { Feasible } \\
\text { completeness }\end{array}$ & $\begin{array}{l}\text { Formal semantics } \\
\text { Modifiability } \\
\text { Analyzability }\end{array}$ & $\begin{array}{l}\text { Driving questions } \\
\text { Model reuse } \\
\text { Model testing and } \\
\text { consistency } \\
\text { checking }\end{array}$ \\
\hline Pragmatic & $\begin{array}{l}\text { Feasible } \\
\text { comprehension }\end{array}$ & $\begin{array}{l}\text { Operational semantics } \\
\text { Executability }\end{array}$ & \begin{tabular}{|l} 
Inspection \\
Visualization \\
Filtering \\
Rephrasing \\
Paraphrasing \\
Explanation \\
Execution \\
Animation \\
Simulation
\end{tabular} \\
\hline $\begin{array}{l}\text { Perceived } \\
\text { semantic }\end{array}$ & $\begin{array}{l}\text { Feasible } \\
\text { perceived validity } \\
\text { Feasible } \\
\text { perceived } \\
\text { completeness }\end{array}$ & Variety & Participant training \\
\hline Social & $\begin{array}{l}\text { Feasible } \\
\text { agreement }\end{array}$ & Inconsistency handling & $\begin{array}{l}\text { Model integration } \\
\text { Conflict resolution }\end{array}$ \\
\hline
\end{tabular}

As already mentioned, many means might be useful on several levels, and we have here positioned them together with the goal where they are believed to have the most effect. This part of the framework is summarised briefly in Table 1. 
A more detailed and formal description of the framework can be found in (Krogstie 1995; Krogstie and Sølvberg 2000) where several modelling approaches both commercially available and academic have been evaluated. The framework has earlier been used for evaluation purposes for workflow modelling languages, SQFD, a requirement specification technique, and UML in (Carlsen et al. 1997; Krogstie 1999; Krogstie 2001) respectively. We discuss below how the framework can be specialised for requirements specification models.

\section{SPECIALIZING THE QUALITY FRAMEWORK FOR REQUIRMENTS SPECIFICATION MODELS}

In (Davis et al. 1993) the work on quality properties for a software requirement specification (SRS) up until that time is summarised and extended. The paper also includes proposals for metrics and weights for the different properties. The following properties are discussed:

- Unambiguous

- Complete

- Correct

- Understandable

- Verifiable

- Internally Consistent

- Externally Consistent

- Achievable

- Concise

- Design-independent

- Traceable

- Modifiable

- Electronically Stored

- Executable/Interpretable/Prototypable

- Annotated by Relative Importance

- Annotated by Relative Stability

- Annotated by version

- Not Redundant

- At Right Level of Detail

- Precise

- Reusable

- Traced

- Organised

- Cross-referenced 
An SRS can be looked upon either as being a model of the perceived future IS as required by someone, or of the perceived future CIS without locking it to one specific implementation. In either case, the domain also includes the goal of the task or project in which the requirement specification is a sub-task, statements about the resource constraints for this particular development effort, and already base-lined documents and models created earlier in the development effort. In more detail, the domain for an SRS can be divided into:

- Primary domain: Everything the CIS is supposed to do (for the moment ignoring the different views the stakeholders have on the CIS to be produced).

- Pre-existing context: Constraints on the model because of earlier baselined models such as business requirements and statements of work.

- Purpose context: Constraints through the fact that one wants to produce a CIS based on the software requirement specifications under given time and resource constraints.

In an SRS, one usually use a mix of conceptual models and natural language text, thus it is necessary to include quality means for both kinds of representation types in combination. Thus in addition to our own work and the work of Davis, we base the overview of work done on quality for textual requirements models (Fabbrini et al. 1998) One important aspect when discussing requirements models, is that they are meant to be understood by persons with very varying background (compared to e.g. design models which are normally only used by persons with detailed software development knowledge).

We discuss means within each quality level in detail, starting with those areas that are specifically mentioned by Davis. We have highlighted these properties in boldface when positioning them within the quality framework below.

\subsection{Physical quality of an SRS}

The basic quality features on the physical level is externalisation, that the knowledge of some social actor has been externalised by the use of a modelling language, and internaliseability, that the externalised model is persistent and available enabling the audience to make sense of it. The only property in this area mentioned by Davis is that the SRS should be electronically stored. This is subsumed by the persistence mean for addressing the physical quality aspect of internaliseability.

Important means for achieving this are using modelling languages that are appropriate for the domain and participant knowledge as discussed above under language quality. An important aspect in relation to a requirement 
specification is that the languages used should not put too high constraints on the technical solution. Properties in the discussion of Davis that potentially influence this area are: Design independent, Traceable, Annotated by relative importance, Annotated by relative stability, Annotated by version, and Precise. Since these properties are not always covered and mandated as an integral part of the modelling language, we will return to these areas in more detail as part of semantic quality. One important activity in this area is the adaptation of the meta-model of the language used to suit the domain and those modelling. This involves either adding concepts, but also removing or hiding concepts (temporarily) from the language if they are not relevant for the modelling of the particular domain.

Internaliseability on the physical level has two primary means, persistence and availability: Many of the general activities in connection with physical quality are based on traditional database-functionality using a repository-solution for the long-term internal representation of the model. In addition, it is regarded necessary for advanced tools for modelling and requirement specification to include functionality such as version control and configuration management, that is not normally found in conventional DBMSs.

\subsection{Empirical quality of an SRS}

Davis partly addresses this area with the property understandable: An SRS is understandable if all classes of SRS readers can easily comprehend the meaning of all requirements with a minimum of explanation. An important factor in achieving this is language quality aspects of the modeling languages that have been used such as comprehensibility appropriateness. The property Concise (An SRS is concise if it is as short as possible without affecting any other quality of the SRS) is a mean at this level, often being related to the expressive economy of the language being used. It can also be linked to overall size limitation of the SRS. To further make Davis' goal more concrete, the same guidelines apply as for diagrammatical or textual models in general when it comes to empirical quality. For informal textual models, a range of means for readability has been devised, such as a number of different readability indexes. For computer-output specifically, many of the principles and tools used for improved human-computer interface are relevant at this level. Other general guidelines regard not mixing different fonts, colours etc. in a paragraph being on the same level within the overall text. Another set of techniques which is often useful here, are those devised within so-called information theory. For graphical models in particular, layout modifications are found to improve the comprehensibility of models. 


\subsection{Syntactic quality of an SRS}

There is one syntactic goal, syntactical correctness, meaning that all statements in the model are according to the syntax and vocabulary of the language. Syntactic errors are of two kinds; Syntactic invalidity, in which words or graphemes not part of the language are used and syntactic incompleteness, in which the model or text lacks constructs or parts to obey the language's grammar. This area is not addressed directly by Davis, although some of the aspects on the semantic level can easily be reduced to syntactic issues by mandating certain aspects to be part of the language (e.g. priority, version, and stability information, see below). Since Davis does not mention this explicitly as a mean, we will discuss these properties as part of semantic quality.

The main mean on this level is the use of a language with formal syntax (also mentioned as a mean by Davis), in which case it can be possible to provide different types of tool support to achieve syntactic quality. To assure the syntactic quality of the model, syntax checks should be provided as an integral part of the modelling support. The checks may be carried out along two main directions.

1. Error prevention, adapting the principle of syntax-directed editors, making it impossible to make certain kinds of syntactic errors.

2. Error detection, with explicit checking being enforced by the user and the check both detects and report existing errors, or also suggest how to correct the error.Errors due to syntactical incompleteness usually have to be checked in this fashion.

(Fabbrini et al, 2001) discusses more detailed means on the syntactical and structural level, focussing on purely textual SRS-documents.

\subsection{Semantic quality of an SRS}

Most of the properties discussed by Davis concern semantic quality. It is important to notice that these quality properties have been suggested under the assumption of an objectivistic world-view. When comparing them with validity and completeness as we have defined them, we thus do this under the presumption that the modelling domain is inter-subjectively agreed among the social actors.

First, when looking upon semantic quality relative to the primary domain, we have the property Complete. According to Davis, an SRS is complete if

1. Everything that the software is supposed to do is included in the SRS

2. Responses of the software to all realisable classes of input data in all recognisable classes of situations are included. 
3. All pages are numbered, all figures and tables are numbered, named, and referenced; all terms are defined; all units of measure are provided; and all referenced material are present.

4. No sections are marked "To be determined".

The first point is the same as our measure of completeness, whereas achieving the second can be supported through using the so-called driving question technique. Item three and four on the other hand is a kind of incompleteness that potentially is easier to deal with. This can be done either by manually checking for such situation after including them as part of a standard document-structure to be followed for the SRS, or by including such aspects as part of the syntax of the modelling language. In this way, one is able to reduce what is presented as a semantic problem into one of checking for syntactic completeness and validity at the potential cost of restricting the freedom in the expression of requirements.

Correct: An SRS is correct if and only if every requirement represent something required of the system to be built. This is the same as what we term 'validity'.

The property internally consistent (An SRS is internally consistent if and only if no subset of individual requirements stated therein conflict) is subsumed by the combination of validity and completeness since an inconsistency must be caused by at least one invalid statement or the lack of a statement that are to sort out the inconsistency. To illustrate, consider the case in which you model an organisation's business rules for implementing these in an information system. Suppose the system must account for the following two rules:

- "If a company has been our customer for more than 10 years, the customer status should be 'high priority"'

- "If a customer has been late with payments more than three times, the customer status should be 'low priority"'.

What then if a company who has been the customer for 12 years is late with payments more than three times? You could decide to change the first rule to rate the customer as 'medium priority' or the second to "more than four times". Either of these two actions would mean that the original rules were invalid. On the other hand, you might decide that both rules are valid, and add another rule "if there are contradictory rules about customer status, the sales manager should resolve the issue", which requires the system simply to notify someone. Adding a rule to resolve a contradiction would mean that the original model was incomplete because it had no such rule.

Davis suggests using languages with formal syntax and semantics to address inconsistency, a mean for semantic quality also proposed in our framework. Note that semantic consistency checking might only detect 
inconsistencies, it will be up to human judgement if the inconsistency is because of invalidity or incompleteness of the model.

Another property being either a matter of incompleteness or invalidity relative to the primary domain is precise (An SRS is precise if and only if (a) numeric quantities are used whenever possible and (b) the appropriate levels of precision are used for all numeric quantities). The first aspect is covered by completeness. If the granularity of precision is too high, this can also be regarded as incompleteness, whereas if it is too low, there is a case of invalidity.

Properties related to the pre-existing context are:

- Traced (An SRS is traced if and only if the origin of each of its requirements is clear) is subsumed by completeness since such links to other models and/or sources of the requirements should be captured in the model if they are deemed relevant.

- Externally consistent (An SRS is externally consistent if and only if no requirement stated therein conflict with any already base-lined project documentation). Statements within such documentation will be part of the pre-existing context; thus, the same can be said about external consistency as was said about internal consistency above.

The following properties are related to completeness and validity relative to the purpose context.

- Annotated by Relative Importance: An SRS is annotated by relative importance if a reader can easily determine which requirements are of most importance, which are next most important etc. Since this is usually needed to be able to allocate resources sensibly, and determine priorities when budgets are inadequate, this is part of completeness.

- Annotated by Relative Stability: An SRS is annotated by relative stability if a reader can easily determine which requirements are most likely to change, which are next most likely etc. Since this is needed for designers to know where to build in flexibility, an SRS that is not annotated in this way is incomplete.

- Annotated by Version: An SRS is annotated by version if a reader can easily determine which requirements will be satisfied in which version of the product. When relevant, the lack of this information is also an example of incompleteness.

On the above three aspects, if it is decided that the language for modeling being used should contain such information (e.g. priority information using QFD (Krogstie 1999) or deontic operators in a rule language (Krogstie and Sindre 1996)), the lack of this can rather be looked upon as an example of syntactic incompleteness than semantic incompleteness.

- Traceable: An SRS is traceable if and only if it is written in a manner that facilitates the referencing of each individual statement. This indicates 
requirements to the language to be used for modelling, thus if the decided language include these kind of aspect, a requirement specification missing them would be syntactically incomplete. If these aspects are not formally included in the language, one needs to treat them as problems of semantic completeness.

- Verifiable: An SRS is verifiable if there exist finite, cost effective techniques that can be used to verify that every requirement stated therein is satisfied by the system to be built. This is partly related to completeness, especially when the requirement is difficult to verify because of ambiguity (see also below). Problems with verifiability because of lack of precision are discussed under the property 'precise'. When verifiability is problematic because of undecidability, this should be explicitly stated if it is relevant.

- Achievable: An SRS is achievable if and only if there could exist at least one system design and implementation that correctly implements all the requirements stated in the SRS. Since it is part of the purpose of an SRS that it should be transformed (usually manually) into a computerised information system, an SRS that is not achievable is invalid. This specific kind of invalidity calls for particular means such as establishing proof of concept through technologically oriented prototyping.

- Design-independent: An SRS is design-independent if and only if there exist more than one system design and implementation that correctly implements all requirements stated in the SRS. This is covered by validity, since if the SRS was not design-independent, it would be overconstrained, and these extra constraints can be looked upon as invalid statements in an SRS.

- At right level of detail: Requirements can be stated at many levels of abstractions. The right level of detail is a function of how the SRS is being used. Generally, the SRS should be specific enough so that any system built that satisfies the requirements in the SRS satisfies all user needs, and abstract enough so that all systems that satisfy all user needs also satisfy all requirements. This indicate that the requirements specification need to be complete, and not over-constrained, i.e. valid, as discussed earlier, thus no new aspects are really included by this property.

- Unambiguous: An SRS is unambiguous if and only if every requirement stated therein has only one possible interpretation. On a high level, this can be claimed to be subsumed by validity and completeness: If the model is consistent and valid, nothing is wrong with having ambiguity, except that you should state explicitly that all alternative interpretations are intended. Without this explicit statement, there is incompleteness related to the purpose context. This said, an unambiguous specification is 
obviously better then an ambiguous from the pragmatic point of view, even if every interpretation of the ambiguity in itself is correct. For instance, a reader may at some point not recognise the ambiguity and consider one interpretation only. Davis suggest the use of formal languages to address ambiguity, similarly to us indicating that using modelling languages with a formal semantics is a mean for achieving semantic quality. Many ambiguities can also be detected on a syntactic level (see e.g. (Fabbrini et. al. 2001), but similarly as for inconsistency, what is the right interpretation must be decided on the semantic levels by persons.

Some additional semantic means mentioned as properties by Davis are:

- Modifiable: An SRS is modifiable if its structure and style are such that any changes can be made easily, completely and consistently. To improve the semantic quality of a model, one needs to change the model. This includes both the cases where the model is found invalid or incomplete in relation to a stable domain, or when the domain changes, e.g. when the requirements to the system or its environment change. In connection to this, Davis suggests the property Not redundant (An SRS is redundant if the same requirement is stated more than once). Unlike the other properties, redundancy is not necessarily bad. Redundancy can in fact improve pragmatic quality (see below). The main problem of redundancy hits when the SRS is changed. Thus, avoiding uncontrolled redundancy is a (secondary) mean to ensure modifiability.

\subsection{Pragmatic quality of an SRS}

The main goal of pragmatic quality is comprehension. There are several reasons to emphasise comprehension, and not comprehensibility at this level. First, the ultimate goal is that the model is understood, not that it is understandable. Comprehensibility as such was discussed as part of empirical quality, whose achievement acts as a possible mean to achieve pragmatic quality. Some other properties mentioned by Davis are:

- Executable/Interpretable/Prototypable: An SRS is executable, interpretable, or prototypable if and only if there exists a software tool capable of inputting the SRS and providing a dynamic behavioural model. To perform the indicated activities, one obviously need tool support for models developed in languages having an operational semantics which can be interpreted by a tool, although the existence of the tool support is not a quality feature of the model itself.

- Organised: An SRS is organised if and only if its contents are arranged so that readers can easily locate information and logical relationships among adjacent sections are apparent. One way is to follow any of many SRS 
standards, e.g. group by type of requirement, class of user, common stimulus, common response, feature, or object.

- Cross-referenced: An SRS is cross referenced if and only if crossreferences are used in the SRS to relate sections containing requirements to other sections containing: Identical (i.e. redundant) requirements, more abstract or more detailed descriptions of the same requirements and requirements that depend on them or on which they depend. As discussed earlier, such links are needed to assure the comprehension of the overall model; thus having them can be classified as a pragmatic mean. They are also related to modifiability. Using e.g. hyperlinks and having advanced browsing capabilities are useful tool support in this area.

There are a number of other activities and possible tool-functionality that can support pragmatic quality, such as audience training, inspections and walkthroughs, model rephrasing, model filtering, animation, explanation generation, and simulation. None of these means are discussed by Davis.

\subsection{Social quality of an SRS}

The main goal of social quality is agreement between involved social actors. Davis does not address this area. Tool support in this respect is most easy to device on achieving agreement in models created based on the different internal reality of the participants that are to agree. One can also support the specific process of achieving feasible agreement. Based on this, the main activity for achieving feasible agreement is model integration with specific emphasis on conflict resolution between the models to be integrated. Argumentation tools are also useful at this level.

\subsection{Orthogonal aspects}

Finally, there is one of the properties suggested by Davis that can be looked upon across all the semiotic levels namely Reusable: An SRS is reusable if and only if its sentences, paragraphs, and sections can be easily adopted and adapted for use in subsequent SRS. This is dependent on many factors at different quality levels:

- The model needs to have good physical quality i.e. it must be physically represented in a persistent form that is available to those who potentially will want to reuse it.

- For reuse of semi-formal and formal models, Davis do not expect the actual models to be reusable as is, but rather that their presence will cause the next SRS writer to reuse the use of such modelling languages. For this to be successful, the original models should be syntactically correct. 
- In cases where one actually wants to reuse the model as is (i.e. were the domains are very similar), it should have a high semantic quality. For white-box reuse, the model need to be modifiable, and should also be comprehensible and comprehended, thus one need to support techniques for achieving pragmatic quality. The model should also be annotated with additional statements making it easier to find the sought for model, thus influencing the completeness relative to the purpose context of the model to support an anticipated need for reuse.

- Where existing models need to be compared with models developed in a separate project, social means and techniques such as model integration and conflict resolution can be useful to investigate to what extent the solutions based on the model to be reused, should be reused.

\section{OVERALL COMPARISON AND CONCLUSION}

As also discussed in (Lindland, Sindre, and Sølvberg 1994), the kind of overviews as those presented by Davis has some weaknesses. We see that still, the properties are partly overlapping. Modifiability is for instance related to redundancy, traceability, machine readability, tracedness, and that the specification is organised and cross-referenced. The problem appears partly because the list mixes goals and means to achieve these goals and because some goals are unrealistic, even impossible to reach. According to the first definition of complete, for example, a specification should include everything that the software is supposed to do. This is addressed in our framework with the notion of feasibility (related to the purpose context). It is indirectly addressed also by Davis by giving suggestions on standards for an SRS through other of his properties, although he is not linking these up to the discussion on completeness.

Other important points when comparing the frameworks are:

- Whereas Davis' work can be classified as objectivistic, built on the belief that it is possible to state true, objective requirements to a CIS, we take into account that the requirements to a CIS are constructed as part of the dialogue between the involved participants. In this light, areas such as consistency and validation become more complex.

- We are able to discuss the relationships between the knowledge, models, and understanding of the individual participants of the modelling effort, and not only the relationships between the abstracted "need of the customers" and the model in the form of an SRS on an aggregated level.

- The technical areas (physical, empirical and syntactic quality) are surprisingly poorly covered by Davis. For pragmatic quality, only some 
of the many possible means described in the modelling literature are mentioned.

- Davis does not discuss social quality and agreement. Reaching agreement is by (Pohl 1994) regarded as one of the three main dimensions of a requirement specification process.

- Our framework is meant to be a general framework for the assessment of quality of models in general. This is also its main weakness, since it by itself is rather abstract and difficult to apply in practical work. By including the properties of requirement specifications within the framework as a specialisation of the framework for this specific model type, we get the best from two worlds. This is specifically apparent in the area of semantic quality, where the work reported by Davis helps us to develop a much more detailed description.

There are several interesting paths for further work on the framework. Primarily we are pursuing the development of process guidelines based on applying the framework as has been partly described earlier in (Krogstie and Sølvberg 2000; Sindre and Krogstie 1995). The steps in this process have been related to existing RE-processes (Loucopoulos and Karakostas 1995). This work has been omitted from this paper due to size limitations.

We are also developing additional product metrics based on the work by (Davis et al 1993) but rather than using this to come up with a total weighted average, subsets of these metrics corresponding to the different quality levels will be useful in the overall RE-process. Similar work should be attempted regarding the extension of the framework for other types of models. Metrics in connection to the use of specific modelling languages and domains should also be developed based on existing work (Costello and Liu 1995; Fabbrini et al. 2001;Hommes and van Reijswoud 1999; Jaffe 1991; Moody and Shanks 1994).

Finally, we note that we have in this paper basically looked upon the individual statements of the SRS, approaching the problem area of quality of an SRS using a sign-oriented semiotics. Another approach is to look at the illocution of the SRS as a whole (Janson and Woo, 1995). For this we would probably like to utilise a text-semiotic theory rather than the sign-oriented semiotics which is the basis for the semiotic ladder.

\section{REFERENCES}

Berger, P. and Luckmann, T. (1966) The Social Construction of Reality: A Treatise in the Sociology of Knowledge. Penguin

Carlsen, S. Krogstie, J. Sølvberg, A. and Lindland, O. I. (1997) Evaluating Flexible Workflow Systems, in Nunamaker, J. F. and Sprague, R. H. (eds.) Proceedings of the Thirtieth 
Annual Hawaii International Conference on System Sciences HICCS'97. Volume II Information Systems- Collaboration Systems and Technology Hawaii, January

Costello, R. J. and Liu, D-B. (1995) Metrics for requirements engineering. Journal of Systems and Software, 29(1):39-63, April

Dahlbom, B. (1991) The idea that reality is socially constructed in Floyd, C., Zullighoven, H., Budde, R., and Keil-Slawik, R. Software Development and Reality Construction Springer 101-126

Davis, A. M. Overmeyer, S. Jordan, K. Caruso, J. Dandashi, F. Dinh, A. Kincaid, G. Ledeboer, G. Reynolds, P. Sitaram, P. Ta, A. and Theofanos, M. (1993) Identifying and measuring quality in a software requirements specification. In Proceedings of the First International Software Metrics Symposium pages 141-152.

Fabbrini, F., Fusani, M., Gervasi, V., Gnesi, S., Ruggieri, S. (1998) Achieving Quality in Natural Language Requirements, in Proceedings of the 11th International Software Quality Week (QW'98) San Francisco, California, USA 26-29 May

Fabbrini, F., Fusani, M., Gnesi, S. and Lami, G. (2001) An Automatic Quality Evaluation for Natural Language Requirements, Proceedings of REFSQ'01, 4-5 June, Interlaken, Switzerland.

Falkenberg, E. D. et al. (1996) (eds.) A framework of information systems concepts. The FRISCO Report, December

Engeström, Y. et al. (1999) (eds.), Perspectives on Activity Theory. Cambridge University Press.

Hommes, B-J. and Reijswoud, V van. (1999) The Quality of Business Process Modeling Methods. in Falkenberg, E. D., Lyytinen, K. and Verrijn-Stuart, A. A. (eds.) Proceedings of the IFIP8.1 working conference on Information Systems Concepts (ISCO4); An Integrated Discipline Emerging. Leiden, Netherlands, 20-22 September.

Jaffe, M. et al. (1991) Software requirements analysis for real-time process-control systems. IEEE Transactions on Software Engineering 17(3): 241-258, March.

Janson, M.A., and Woo,C. (1995) Comparing IS Development Tools and Methods: Using In Speech Act Theory, Information and Management, Vol.28, No.1, 1-12.

Krogstie, J. (1995) Conceptual Modeling for Computerized Information Systems Support in Organizations. Ph.D. Thesis IDT, NTH, Trondheim, Norway.

Krogstie, J. Lindland, O. I. and Sindre, G. (1995a) Defining quality aspects for conceptual models, in Falkenberg, E. D. Hesse, W. and Olive, A. (eds.) Proceedings of the IFIP8.1 working conference on Information Systems Concepts (ISCO3); Towards a consolidation of view pp. 216-231 Marburg, Germany, March 28-30.

Krogstie, J. Lindland, O. I. and Sindre, G. (1995b) Towards a deeper understanding of quality in requirements engineering. in livari, J. Lyytinen, K. and Rossi, M. (eds.). Proceedings of the 7th International Conference on Advanced Information Systems Engineering (CAiSE'95) pages 82-95, Jyväskylă, Finland.

Krogstie, J. and Sindre, G. (1996) Utilizing Deontic Operators in Information Systems Specification. Journal of Requirements Engineering, 4(1) pp 210-237.

Krogstie, J. and Sølvberg, A. (2000), Information Systems Engineering - Conceptual Modeling in a Quality Perspective, Draft of Book, Information Systems Groups, NTNU, Trondheim, Norway.

Krogstie, J. (1999) Using Quality Function Deployment in Software Requirements Specification, in Opdahl, A., Pohl, K. and DuBois, E. (eds.). Proceedings of the Fifth International Workshop on Requirements Engineering: Foundations for Software Quality (REFSQ'99), pages 171-187, Heidelberg, Germany 14-15 June 
Krogstie, J. (2001) Using a Semiotic Framework to Evaluate UML for the Development of Models of High Quality, in Keng Siau, Terry Halpin (eds.) Unified Modeling Language: Systems Analysis, Design and Development Issues, Idea Group

Lindland, O. I. Sindre, G. and Sølvberg, A. (1994) Understanding quality in conceptual modeling. IEEE Software, pages 42-49, April

Loucopoulos, P. and Karakostas, V. (1995) System Requirements Engineering. McGraw-Hill.

Moody, D. L. and Shanks, G. G. (1994) What makes a good data model? Evaluating the quality of entity relationship models, in Proceedings of the 13th International Conference on the Entity-Relationship Approach (ER'94), pages 94-111, Manchester, England.

Nöth, W. (1990) Handbook of Semiotics Indiana University Press

Pohl, K. (1994) The three dimensions of requirements engineering: A framework and its applications. Information Systems, 19(3): 243-258, April.

Schuette, R. (1999) Architectures for Evaluating the Quality of Information Models - A Meta and an Object Level Comparison, in Conceptual Modeling-ER'99 18th International Conference on Conceptual Modeling, 490-505 Paris, November

Sindre, G. and Krogstie, J. (1995) Process Heuristics to Achieve Requirements Specification of Feasible Quality, in Editors Pohl, K., and Peters. P (eds.) Second International Workshop on Requirements Engineering: Foundations for Software Quality (REFSQ'95), Jyvälskylä, Finland, 92-103

Wand, Y. and Weber, R. (1993) On the Ontological Expressiveness of Information Systems Analysis and Design Grammars Journal of Information Systems 3(4) pp. 217-237. 